ARTICLE

\title{
Nuclear spin coupling crossover in dense molecular hydrogen
}

\author{
Thomas Meier (10 ${ }^{1 凶}$, Dominique Laniel ${ }^{2}$, Miriam Pena-Alvarez ${ }^{3}$, Florian Trybel ${ }^{1}$, Saiana Khandarkhaeva ${ }^{1}$,
} Alena Krupp ${ }^{1}$, Jeroen Jacobs ${ }^{4}$, Natalia Dubrovinskaia (i) ${ }^{2} \&$ Leonid Dubrovinsky (i) $^{1}$

One of the most striking properties of molecular hydrogen is the coupling between molecular rotational properties and nuclear spin orientations, giving rise to the spin isomers ortho- and para-hydrogen. At high pressure, as intermolecular interactions increase significantly, the free rotation of $\mathrm{H}_{2}$ molecules is increasingly hindered, and consequently a modification of the coupling between molecular rotational properties and the nuclear spin system can be anticipated. To date, high-pressure experimental methods have not been able to observe nuclear spin states at pressures approaching $100 \mathrm{GPa}$ (Meier, Annu. Rep. NMR Spectrosc. 94:1-74, 2017; Meier, Prog. Nucl. Magn. Reson. Spectrosc. 106-107:26-36, 2018) and consequently the effect of high pressure on the nuclear spin statistics could not be directly measured. Here, we present in-situ high-pressure nuclear magnetic resonance data on molecular hydrogen in its hexagonal phase I up to $123 \mathrm{GPa}$ at room temperature. While our measurements confirm the presence of ortho-hydrogen at low pressures, above $70 \mathrm{GPa}$, we observe a crossover in the nuclear spin statistics from a spin-1 quadrupolar to a spin-1/2 dipolar system, evidencing the loss of spin isomer distinction. These observations represent a unique case of a nuclear spin crossover phenomenon in quantum solids.

\footnotetext{
${ }^{1}$ Bayerisches Geoinstitut, University of Bayreuth, Bayreuth, Germany. ${ }^{2}$ Material Physics and Technology at Extreme Conditions, Laboratory of Crystallography, University of Bayreuth, Bayreuth, Germany. ${ }^{3}$ Centre for Science at Extreme Conditions and School of Physics and Astronomy, University of

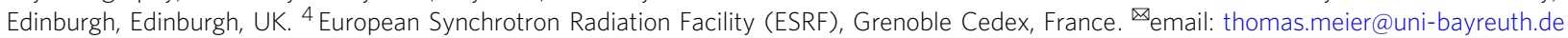


C hanges in electronic spin-statistics under changing thermodynamic conditions are an established physical crossover phenomenon ${ }^{1-3}$. It has direct applications for spintronics ${ }^{4}$ and enables the understanding of the stabilisation of magnetospheres of rocky (Earth-like) planets ${ }^{5}$ as well as gas- and ice-giants ${ }^{6}$. The degrees of freedom of the nuclei spins, however, are widely approximated as fixed within the analysis of experiments, due to large shielding by core electrons and the extremely short atomic distances necessary to induce such fundamental changes.

Hydrogen, on the other hand, exhibits no core electrons and when bound contributes its electron to the molecular bond. Furthermore, due to the low mass of the hydrogen nuclei, quantum nuclear effects are considered to be significantly more pronounced compared to all other elements. The combination of both effects results in a number of fascinating physical phenomena in molecular $\mathrm{H}_{2}{ }^{7-9}$.

One property intrigued physicists in particular: the nature of the nuclear spin of the $\mathrm{H}_{2}$ molecule and the resulting coexistence of the spin isomers ortho- (ortho- $\mathrm{H}_{2}$ ) and para-hydrogen (para- $\mathrm{H}_{2}$ ). Following Pauli's exclusion principle, in order for the total $\mathrm{H}_{2}$ molecular wave function to be antisymmetric under exchange of atomic positions, demands for the rotational ground state $J=0$, that the corresponding total nuclear wave function is antisymmetric (singlet state of $I=0$, i.e. para- $\mathrm{H}_{2}$ ). Analogously, for the $J=1$ rotational state, the total nuclear wave function is required to be symmetric (triplet state of $I=1$, i.e. ortho- $\mathrm{H}_{2}$ ). Therefore, the spin allotropic isomerism of the $\mathrm{H}_{2}$ molecule originates in the coupling of both rotational state and nuclear spin. It has been argued ${ }^{10,11}$ that at high pressure $(P)$ ortho- and para-hydrogen spin isomers remain stable up to the dissociative Wigner-Huntington transition at $P>400 \mathrm{GPa}^{12,13}$.

This can only be assumed for weak or moderate intermolecular interactions, i.e. when nearest neighbour distances $\left(r_{\mathrm{n}}\right)$ are much shorter $(\approx 0.7 \AA$ at ambient conditions $)$ than next-nearest neighbour distances $\left(r_{\mathrm{nn}} \approx 3 \AA\right.$ at ambient conditions), allowing for sufficient intramolecular wave function overlap (left side of Fig. 1a).

Under high enough densities, however, intermolecular interactions increase significantly as $r_{\mathrm{nn}}$ decreases by $\sim 70 \%$ within 100 $\mathrm{GPa}^{14,15}$. At these pressures, $r_{n n}$ approaches $r_{n}$ and collective nuclear quantum fluctuations increase rapidly ${ }^{16}$.

For decades, theoretical ${ }^{8,17,18}$ and experimental ${ }^{9}$ studies indicated that under such extreme pressures odd values of $J$ become unstable, rapidly decaying into even states, which leads to potential indistinguishability of the hydrogen spin isomers on experimental timescales.

The only experimental technique in high-pressure research to directly study the $\mathrm{H}_{2}$ nuclear spin states is nuclear magnetic resonance (NMR) spectroscopy, detecting the linear response of the nuclear spin system upon radio-frequency excitation in a magnetic field $B_{0}$. The spin-singlet state of para-hydrogen is NMR
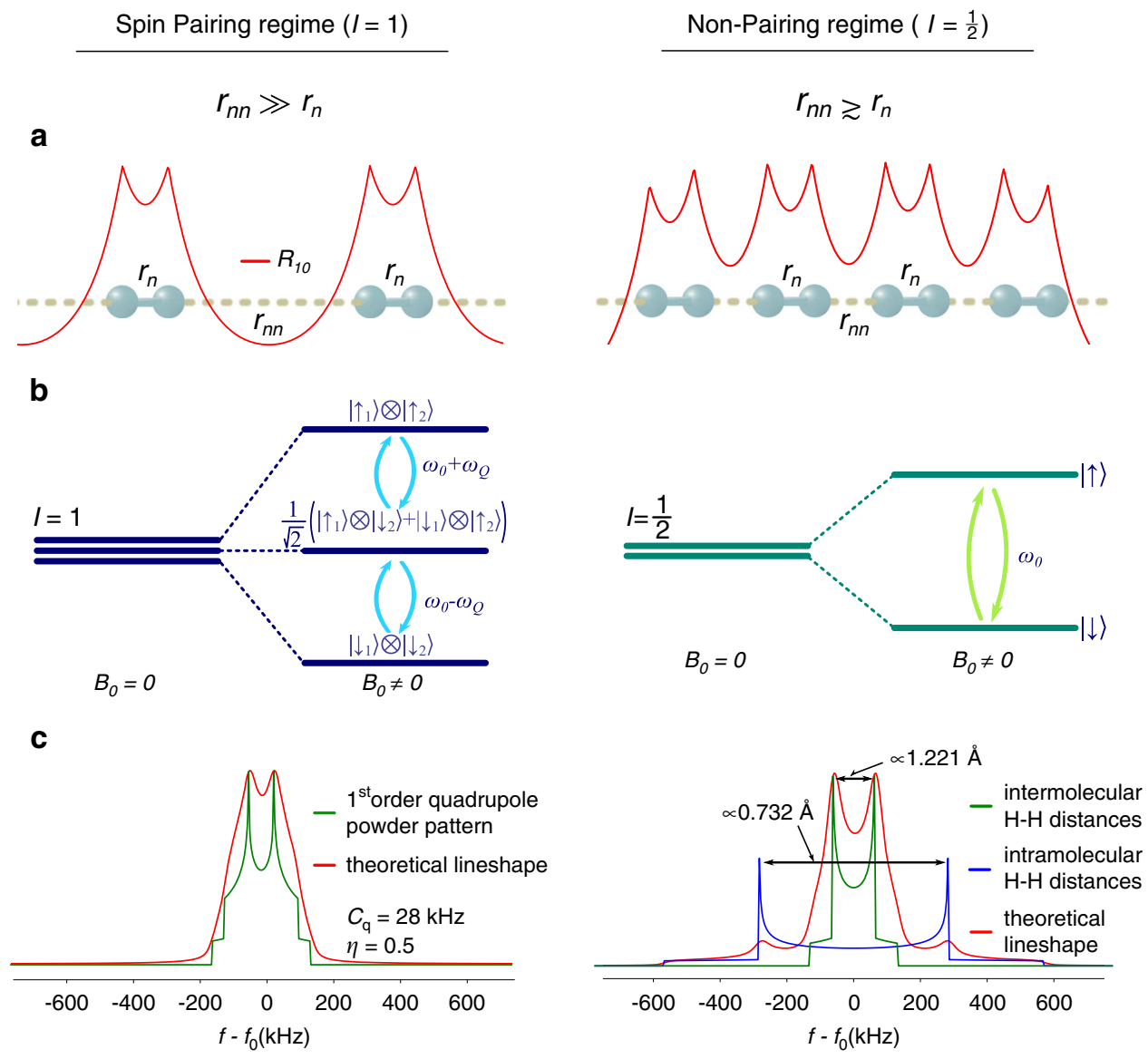

Fig. 1 Overview of both spin-pairing and non-pairing regimes. a Schematic representation of the wave function overlap (red lines) of $\mathrm{H}_{2}$ molecules. b Schematic representation of the nuclear spin energy levels under the influence of an external magnetic field $B_{0}$ for the pairing (i.e. quadrupole interaction) and non-pairing (dipole-dipole interaction) regimes. c Theoretical line shapes in the pairing and non-pairing regime. $\omega_{0}=2 \pi f_{0}$ denotes the Larmor frequency of the hydrogen nuclei, $\omega_{\mathrm{Q}}, \eta$ and $C_{\mathrm{q}}$ are the quadrupole frequency, the asymmetry parameter and the quadrupole coupling constant, respectively. 
silent, whereas application of $B_{0}$ lifts the three-fold degeneracy of the ortho states and allows for an excitation of nuclear spin transitions (Fig. 1b). Nuclear spin-pairing in ortho-hydrogen leads, furthermore, to a finite electric quadrupole moment, $e Q$, interacting with the local charge distribution defined by the structural arrangement of hydrogen molecules. Thus, the quadrupolar coupling can be considered the dominant spin interaction, resulting in characteristic NMR line shapes ${ }^{19}$ (Fig. 1c).

Here, we present ${ }^{1} \mathrm{H}-\mathrm{NMR}$ data of dense molecular hydrogen up to $123 \mathrm{GPa}$ at room temperature and find a distinct crossover in the nuclear spin statistics of molecular hydrogen indicating a loss of ortho-para spin isomer distinction. Details on experimental conditions, spectral simulations, as well as data analysis, are provided in the Methods section.

\section{Results}

Two NMR-DACs equipped with diamond anvils of 250 and $100 \mu \mathrm{m}$ culets were loaded with molecular $\mathrm{H}_{2}$. At low pressure (below $60 \mathrm{GPa}$ ), intense ${ }^{1} \mathrm{H}$ resonances of roughly $500 \mathrm{kHz}$ width were detected. With increasing $P$, the resonance signals broadened significantly approaching $750 \mathrm{kHz}$ at $68 \mathrm{GPa}$ (Fig. 2). Above $68 \mathrm{GPa}$, we observed a resonance narrowing accompanied

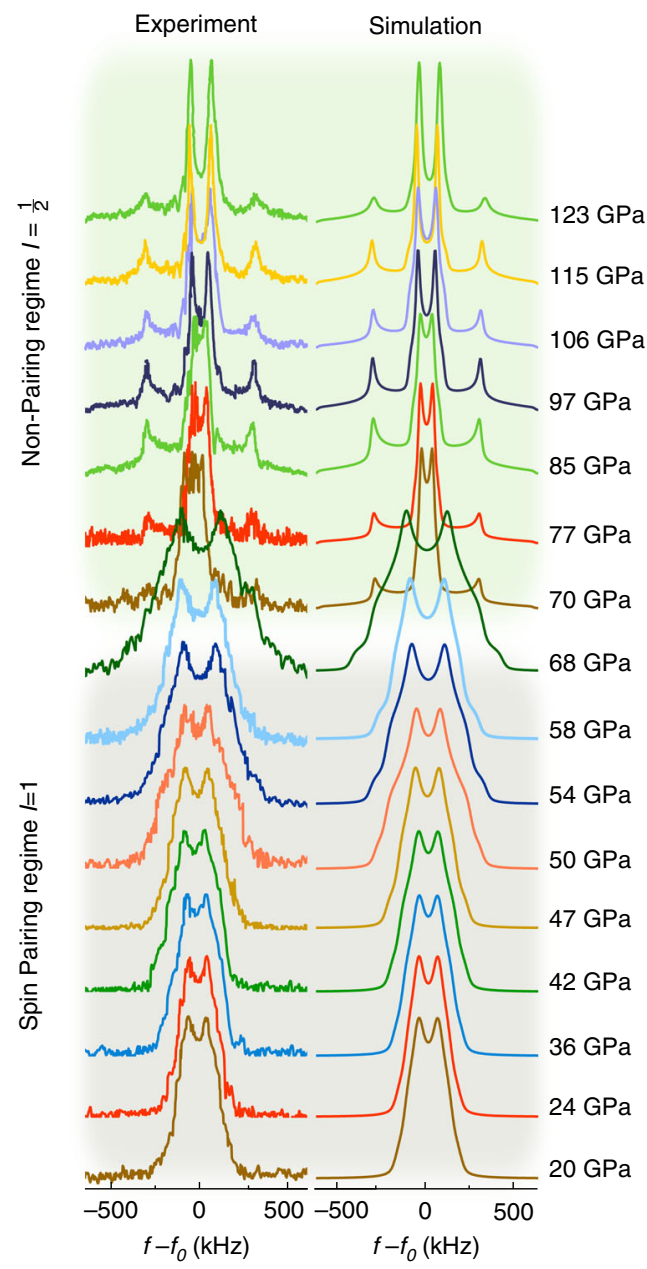

Fig. 2 Experimental and calculated 1H-NMR spectra of molecular H2 up to $123 \mathrm{GPa}$ at room temperature. Between 20 and $68 \mathrm{GPa}$, first-order quadrupole interactions describe the experimental data reasonably well. At $P>68 \mathrm{GPa}$, spectra were found to be broadened by dipole-dipole interaction resulting in a superposition of two Pake doublets corresponding to nearest and next-nearest hydrogen distances. by the emergence of two Pake doublets ${ }^{20}$ with increasing splitting upon compression.

For the quadrupolar nature expected for ortho- $\mathrm{H}_{2}(I=1)$, the electric field $V(\boldsymbol{r})$, defined by the local charge distribution based on the crystal structure of phase I, should influence the shape of the observed resonance lines. Calculated line shapes for an $I=$ 1 spin system are shown in Fig. 2 at pressures of up to $68 \mathrm{GPa}$. The order of magnitude of the quadrupolar interaction energy was considered small relative to the nuclear Zeeman energy ${ }^{19}$ and consequently treated as a first-order perturbation (see "Methods" section for computational details). Up to $68 \mathrm{GPa}$, the measured ${ }^{1} \mathrm{H}-\mathrm{NMR}$ spectra are well described by calculated line shapes broadened by first-order quadrupole interaction. The line shape is mainly controlled by two parameters: (i) the quadrupole coupling constant $C_{\mathrm{q}}$ describing the coupling between $e Q$ and $V(\boldsymbol{r})$ as well as (ii) the electric field gradient asymmetry parameter $\eta$ accounting for the geometry of $V(\boldsymbol{r})$.

Figure $3 \mathrm{a}$ (top panel) shows estimated values of $C_{\mathrm{q}}$, which increase from $28.1(6) \mathrm{kHz}$ at $20 \mathrm{GPa}$ to $61.9(7) \mathrm{kHz}$ at $58 \mathrm{GPa}$. This increase is likely originated in the high compressibility and rapidly reducing next-nearest neighbour distances between molecular $\mathrm{H}_{2}$ units, enhancing quadrupolar coupling. The asymmetry parameter $\eta$ (Fig. 3a, bottom panel) was found to be almost constant within experimental errors varying between 0.44 (6) at $20 \mathrm{GPa}$ and $0.49(9)$ at $58 \mathrm{GPa}$. Based on the hexagonal crystal structure of phase $\mathrm{I}^{14,21}, \eta$ can be expected to be close to 0.5 , which is in excellent agreement with values derived from the analysis of our NMR measurements.

Above $P \approx 68 \mathrm{~Pa}$, however, we observed a sudden decrease in both $C_{\mathrm{q}}$ and $\eta$ coinciding with a resonance peak splitting. As no structural rearrangement of $\mathrm{H}_{2}$ molecules is reported by diffraction methods ${ }^{21}$ or Raman spectroscopy ${ }^{22}$ at room temperature in this $P$-range, effects based on a modification of the $\mathrm{H}_{2}$ nuclear spin system should be considered.

This pressure indicates a turning point in the behaviour of the ${ }^{1} \mathrm{H}$ spin system, as the observed peak splitting devolves from having dominantly quadrupolar characteristics towards a system controlled by nuclear dipole-dipole coupling, resulting in pronounced $I=1 / 2$ line shapes ${ }^{20}$ with a frequency difference between spectral density function singularities directly correlated to the distances between hydrogen atoms. Considering that in this case both, the interaction with the nearest and next-nearest neighbours will result in a dipolar NMR pattern, respectively, a superposition of signals as shown in Fig. 1c can be expected. Computationally obtained values of nearest and next-nearest neighbour distances ${ }^{15}$ are $r_{n} \approx 0.731 \AA$ and $r_{n n} \approx 1.342 \AA$ and at $120 \mathrm{GPa}$. Calculating the distances from the parameter-set obtained through analysis of the NMR spectra for such a mixed scenario resulted in $r_{\mathrm{n}} \approx 0.727 \AA$ and $r_{\mathrm{nn}} \approx 1.27(8) \AA$ at $123 \mathrm{GPa}$, in excellent agreement with the computational estimates by Labet et al. ${ }^{15}$. Figure 2 shows the comparison between experimental spectra and calculated $I=1 / 2$ line shapes between 71 and 123 $\mathrm{GPa}$. The respective values derived for both nearest (top panel) and next-nearest (middle panel) neighbour distances can be found in Fig. 3b. Additionally, the comparison between the equation of state derived from ab initio computations ${ }^{23}$ and diffraction data ${ }^{14,21}$, along with the unit cell volumes (blue dots) derived from $r_{\mathrm{n}}$ and $r_{\mathrm{nn}}$ extracted from the analysis of the NMR spectra are shown in the bottom panel of Fig. $3 \mathrm{~b}$.

Homonuclear Lee-Goldburg decoupling sequences ${ }^{24}$ have been used to suppress quadrupolar and dipolar line broadening in order to resolve isotropic chemical shifts, $\delta_{\text {iso. }}$. Figure $3 \mathrm{c}$ shows the evolution of $\delta_{\text {iso }}$ : initially decreasing from 8.6 to $5.9 \mathrm{ppm}$ between 20 and $59 \mathrm{GPa}, \delta_{\text {iso }}$ has an inflection point at $\sim 60 \mathrm{GPa}$ and raises under further compression to $22.7 \mathrm{ppm}$ at $123 \mathrm{GPa}$. Comparison with Raman data ${ }^{25}$ suggests that the minimum in $\delta_{\text {iso }}$ coincides 

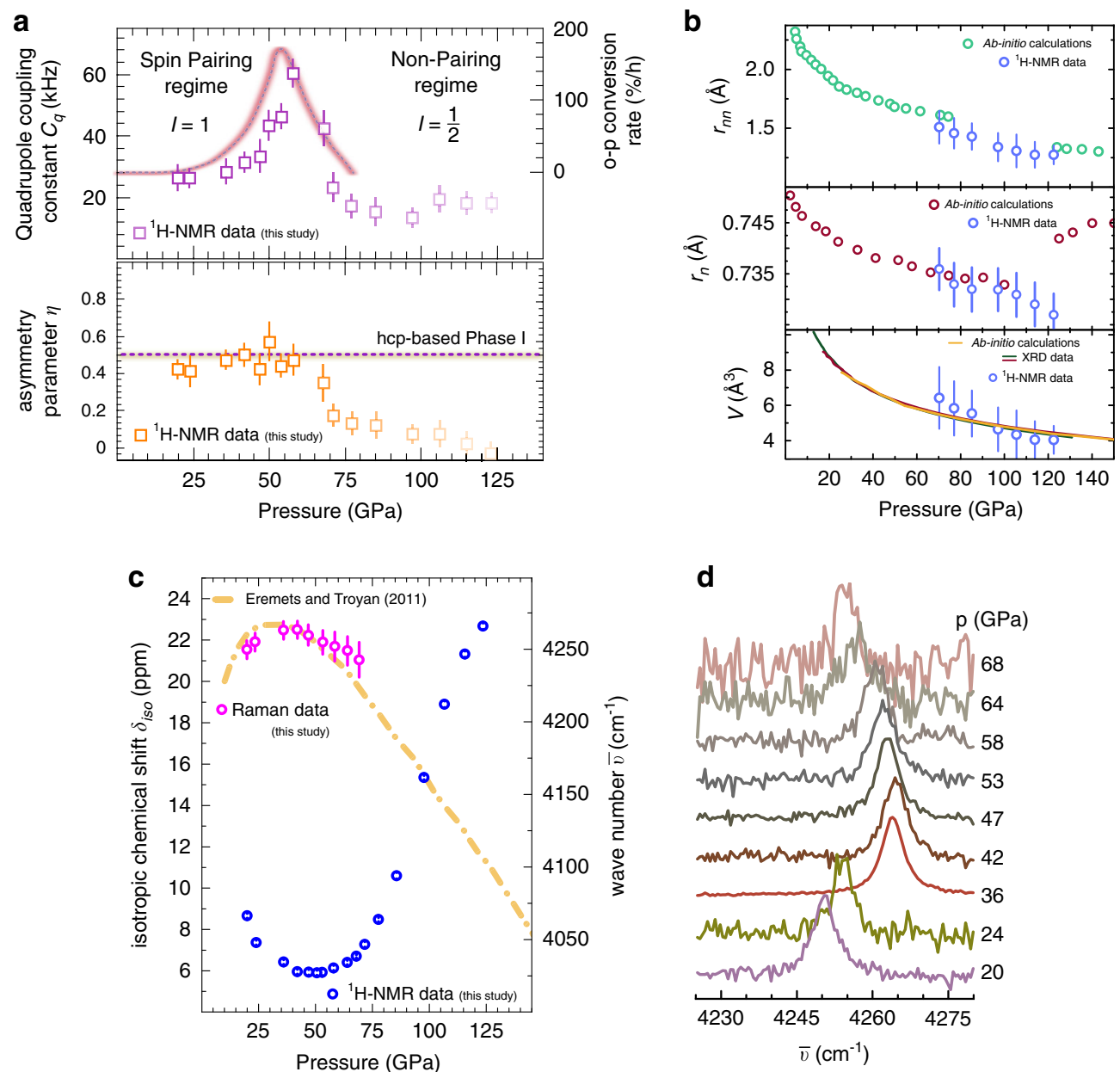

Fig. 3 Extracted ${ }^{1} \mathbf{H}-\mathbf{N M R}$ data of molecular $\mathbf{H}_{\mathbf{2}}$ at pressures up to $123 \mathbf{~ G P a}$ at room temperature. a Top panel: quadrupole coupling constant $C_{q}$ determined from NMR data (squares). The rose line denotes theoretical ortho-para conversion rates from electric quadrupole interaction ${ }^{27}$. Bottom panel: asymmetry parameter $\eta$ in the spin-pairing regime $(P<60 \mathrm{GPa})$. The dashed line represents the inferred $\eta$ based on the hcp structure of Phase I from diffraction experiments. The shading of the squares at $P>60 \mathrm{GPa}$ highlights the crossover to the non-pairing $I=1 / 2$ regime. $\mathbf{b}$ Top panel: next-nearest neighbour distances $r_{\mathrm{nn}}$. Green circles are based on DFT computations ${ }^{15}$. Blue circles are extracted values of $r_{\mathrm{n}}$ and $r_{\mathrm{nn}}$ from the NMR spectra in the nonpairing $I=1 / 2$ regime. Middle panel: nearest neighbour distances $r_{n}$ (blue circles) and DFT calculations ${ }^{15}$ (red circles). The discontinuity at $P>125 \mathrm{GPa}$ in the DFT calculations indicates a transition from the hcp based to a monoclinic structure. Bottom panel: comparison between the equations of state derived from ab initio computations ${ }^{23}$ (yellow line) and diffraction data ${ }^{14,21}$ along with the unit cell volumes (blue dots) derived from $r_{\mathrm{nn}}$ and $r_{\mathrm{n}}$ extracted from the NMR experiments. c Extracted isotropic chemical shift values $\delta_{\text {iso }}$ after homonuclear Lee-Goldberg decoupling. Error bars are within the symbol size. The orange dotted line shows the room temperature Raman shift of the $\mathrm{H}_{2}$ vibron ${ }^{25}$. d Selected Raman spectra of the $\mathrm{H}_{2}$ vibron at increasing pressure. Spectra up to $64 \mathrm{GPa}$ are taken from ref. ${ }^{25}$. Error bars in $\mathbf{a}$ and $\mathbf{b}$ were taken from spectral simulation and comparison with experimental data. Error bars in $\mathbf{c}$ are taken from Voigtian spectral line shape fitting to the experimental data. Error bars of the NMR data after Lee-Goldburg decoupling (blue points in c) were within the symbol size.

with the well-known turn-over in the Raman shift of the $\mathrm{H}_{2}$ vibron caused by a weakening of intramolecular and increased intermolecular interactions ${ }^{26}$.

\section{Discussion}

The presented data analysis leads to the following interpretation of the observed effects: At $P<60 \mathrm{GPa},{ }^{1} \mathrm{H}-\mathrm{NMR}$ data is characteristic for an $I=1$ quadrupolar spin system as expected for ortho- $\mathrm{H}_{2}$. In this regime, individual nuclear spin angular momenta couple with their nearest neighbours (separated on average by $r_{\mathrm{n}}$ ), leading to a significant wave function overlap within the molecular units and a stabilisation of the spin isomers. The excellent agreement between NMR derived values for the electric field gradient asymmetry parameter $\eta$ and values inferred from X-ray diffraction data ${ }^{14,21}$ strengthens this assessment. Following the theoretical study of Strzhemechny et al. $^{27}$, this compression driven enhancement of the quadrupolar coupling constant $C_{\mathrm{q}}$ in this $P$-regime may be interpreted as experimental evidence for the mechanism of orthopara conversion through electric quadrupole interaction.

At $P>60 \mathrm{GPa}$, quadrupolar coupling rapidly diminishes despite the absence of a structural rearrangement of the molecular $\mathrm{H}_{2}$ units. Starting from about $70 \mathrm{GPa}$, spectral features characteristic of homonuclear dipole-dipole coupling between nearest and nextnearest neighbours become apparent. Provided the good agreement between NMR data with $\mathrm{DFT}^{15}$ and experimentally ${ }^{14,21}$ derived intermolecular and interatomic distances, this shift in behaviour implies intramolecular coupling of nuclear spins to become increasingly perturbed. The inflection point in the isotropic chemical shift $\delta_{\text {iso }}$ strengthens this hypothesis as the increasing nuclear de-shielding above $60 \mathrm{GPa}$ indicates a shift of electron density away from individual molecular centres towards intermolecular regions. 
${ }^{14} \mathrm{~N}-\mathrm{NMR}$ on molecular nitrogen at $P=3 \mathrm{GPa}$ (see "Methods" section) supports this argument, as the nitrogen spin system shows clear characteristics of a nuclear spin-triplet state anticipated within the non-pairing regime contrary to the quintuplet state stabilised in the molecular spin-pairing regime.

In this work, in-situ high-pressure nuclear magnetic resonance was used to investigate the nature of the nuclear spin statistics of molecular hydrogen up to $123 \mathrm{GPa}$ in Phase I at room temperature. It was found that even at moderately high pressures $(<100 \mathrm{GPa})$ intramolecular nuclear spin coupling broke down and the hydrogen spin system adopted an average dipolar $I=1 / 2$ value. Crossovers of the nuclear spin statistics of a quantum solid such as hydrogen have so far not been observed and given the large compressibility of hydrogen in conjunction with strong nuclear quantum effects, this crossover phenomenon might only be experimentally observable in molecular $\mathrm{H}_{2}$. Nuclear spin statistics of similar diatomic molecules (e.g. $\mathrm{N}_{2}$ ) are likely to be best described as non-pairing nuclear spins due to enhanced atomic masses as well as reduced compressibilities due to the presence of core electrons.

This nuclear spin-crossover may have far-reaching consequences for understanding different phenomena such as the stabilisation of magnetospheres of gas and ice giant planets containing large quantities of molecular $\mathrm{H}_{2}$.

\section{Methods}

Diamond anvil cell preparation. Two diamond anvil cells, equipped with pairs of diamond anvils with a culets size of 250 and $100 \mu \mathrm{m}$, were prepared. Rhenium gaskets were pre-indented to 25 and $10 \mu \mathrm{m}$, respectively, and 80 and $40 \mu \mathrm{m}$ diameter holes were laser drilled in the centre of the indentation to form the sample cavities, resulting in sample volumes of about 125 and $13 \mathrm{pl}$, respectively.

The diamond anvils were coated with a $1-\mu \mathrm{m}$-thick layer of copper using physical vapour deposition ${ }^{28}$. Double ${ }^{29}$ (in the case of the $250 \mu \mathrm{m}$ diamonds) and triple $^{30}$ (for the $100 \mu \mathrm{m}$ diamonds) stage Lenz-lens radio-frequency resonators were produced by using focused ion beam milling. To ensure electrical insulation and avoid hydrogen diffusion into the rhenium, the gaskets were coated by 500 $\mathrm{nm}$-thick layers of $\mathrm{Al}_{2} \mathrm{O}_{3}$. Radio-frequency excitation coils were made from $100 \mu \mathrm{m}$ thick, Teflon insulated, copper wire and arranged such that a Helmholtz coil pair is formed.

Hydrogen loading was conducted at the ESRF and pressure was increased at cryogenic temperatures to avoid rapid hydrogen diffusion into the diamond anvils. The pressure was calibrated by means of the diamond edge Raman scale ${ }^{31,32}$. Comparison of the vibron frequencies of the $\mathrm{H}_{2}$ samples at elevated pressures shows a slight systematic offset of less than $5 \mathrm{GPa}$ at the highest pressures where Raman data was collected ${ }^{33}$

NMR experiments. All NMR experiments were conducted using a solid-state NMR spectrometer from Tecmag Inc. (Redstone) equipped with a $100 \mathrm{~W}$ pulse amplifier. To polarise the nuclear spin system, we used a sweepable electromagnet with an average magnetic field of $1 \mathrm{~T}$ and sufficiently high homogeneity. Intense ${ }^{1} \mathrm{H}$-NMR signals were recorded at frequencies of $45.26 \mathrm{MHz}$, corresponding to an external magnetic field strength of about $1063 \mathrm{mT}$. Using nutation experiments, we found optimal excitation pulses between 1 and $1.2 \mu$ s for both cells, in reasonable agreement with earlier experiments ${ }^{28-30,34}$.

Free induction decays were excited using a single pulse of $833 \mathrm{kHz}$ to $1 \mathrm{MHz}$ bandwidth. The spectrometer was blanked off for $1 \mu$ s to avoid damage to the preamplifier. Supplementary Figs. S2 and S3 show all ${ }^{1} \mathrm{H}-\mathrm{NMR}$ spectra recorded by this method. 25,000 scans were accumulated for each spectrum (Fig. 2).

In order to resolve isotropic chemical shifts, $\delta_{\text {iso, }}$ a Lee-Goldburg pulse for homonuclear decoupling was used ${ }^{24}$. The resulting narrowed NMR spectra had an FWHM linewidth of about $3 \mathrm{ppm}$, thus the resolution accuracy of $\delta_{\text {iso }}$ was found to be in the order of $0.1 \mathrm{ppm}$ (Supplementary Fig. S4). Resonance frequencies were referenced towards an aqueous solution of tetramethylsilane in a similar DAC at ambient pressure conditions.

Computation of NMR line shapes and asymmetry parameters of the electric field gradient. Calculation of the NMR line shapes was carried out following the analytical method outlined by Bloembergen and Rowland ${ }^{35}, \mathrm{Pake}^{20}$, and Hughes and Harris ${ }^{36}$ :

Using the standard expressions for the resonance frequency distribution $\omega$ for both first-order quadrupole interaction as well as homonuclear dipole-dipole interaction:

$$
\begin{gathered}
\omega(\alpha, \beta, m)=\omega_{Q} \cdot(m+1 / 2) \cdot\left(\frac{3 \cos ^{2} \beta-1}{2}-\frac{\eta}{2} \sin ^{2} \beta \cos (2 \alpha)\right), \\
\omega(\alpha)=d_{i} \cdot\left(\frac{3 \cos ^{2} \beta-1}{2}\right),
\end{gathered}
$$

with

$$
\begin{gathered}
\omega_{Q}=\frac{6 \pi}{2 I(2 I+1)} \cdot C_{\mathrm{q}}, \\
C_{\mathrm{q}}=\frac{e^{2} q Q}{h}, \\
d_{i}=\frac{\mu_{0} \gamma_{n}^{2} \hbar}{8 \pi^{2} r_{i}^{3}},
\end{gathered}
$$

where the Euler angles $\alpha$ and $\beta$ describe the orientation of the crystallites with respect to the external magnetic field. $\gamma_{n}$ is the gyromagnetic ratio of the hydrogen nuclei, $\mathrm{m}$ the nuclear spin quantum number $(m=1,0,-1)$ and $r_{i}$ the average distance between interacting hydrogen nuclei, $r_{\mathrm{n}}$ or $r_{\mathrm{nn}}$, respectively. $\eta$ describes the asymmetry of the electric field gradient tensor $\left(V_{\mathrm{ij}}\right)$ in the principal axis system as:

$$
\eta=\frac{V_{y y}-V_{x x}}{V_{z z}},\left|V_{z z}\right|>\left|V_{x x}\right|>\left|V_{y y}\right| .
$$

The line shape function, $P(\omega)$, for quadrupolar spin interactions, is given by:

$$
P(\omega)=\sum_{m} \int_{-1}^{1} \frac{\mu}{4 \pi} \sin (\beta(\omega, \alpha, m)) \cdot\left(\left\|\frac{\partial \beta(\omega, \alpha, m)}{\partial \omega}\right\|\right) d(\cos (2 \alpha)),
$$

where $\beta(\omega, \alpha, m)$ denotes the inverse function of Eq. (1) with respect to $\beta$, and $\mu$ accounts for the multiplicity of spectral functions. For the dipolar interaction $P(\omega)$, is given by

$$
P(\omega)=\sum_{i} \int_{-1}^{1} \frac{\mu}{4 \pi} \sin \left(\beta\left(\omega_{i}, \alpha\right)\right) \cdot\left(\left\|\frac{\partial \beta\left(\omega_{i}, \alpha\right)}{\partial \omega_{i}}\right\|\right) d(\cos (2 \alpha)),
$$

where $\beta\left(\omega_{i}, \alpha\right)$ denotes the inverse function of Eq. (2) with respect to $\beta$ and $\mu$ accounts for the multiplicity of spectral functions.

Cut-off frequencies of the resulting spectral line functions were chosen according to the Heaberlein convention for NMR shift tensors ${ }^{37}$. Spectral line broadening was accounted for by convolution of the total line shape function with a Voigtian line of defined Lorentzian and Gaussian widths. In order to fit the experimental data, the respective line shape function $P(\omega)$ is optimised by varying $C_{\mathrm{q}}$ and $\eta$ for quadrupolar coupling and $r_{\mathrm{n}}$ and $r_{\mathrm{nn}}$ for dipolar coupling. The corresponding Python scripts are available from the authors upon request. Table 1 summarises all fit parameters.

In order to calculate the asymmetry parameter $\eta$ of the electric field gradient tensor in the spin-pairing regime, we used the second derivative of the electric potential, $V(\boldsymbol{r})$, defined by the molecular centre of gravity positions from diffraction measurements ${ }^{14}$ :

$$
\begin{gathered}
V(\boldsymbol{r})=\frac{e}{4 \pi \epsilon_{0}} \sum_{i} \frac{1}{\sqrt{\left(x-x_{i}\right)^{2}+\left(y-y_{i}\right)^{2}+\left(z-z_{i}\right)^{2}}}, \\
V_{i j}=\frac{\partial V(\boldsymbol{r})}{\partial x_{i} \partial y_{j}} .
\end{gathered}
$$

Using Eq. (6) under consideration of the ordering of the components of $V_{i j}$ in the principal axis system allows the computation of $\eta$ from crystallographic data

${ }^{14} \mathbf{N}-\mathbf{N M R}$ of molecular nitrogen at $3 \mathbf{G P a}$. Molecular nitrogen was measured using natural isotopic composition, where the majority of molecules can be expected to be pairs of ${ }^{14} \mathrm{~N}$ nuclei. As ${ }^{14} \mathrm{~N}$ nuclei have a nuclear spin of $I=1$, one can expect a spin-pairing scenario similar to molecular $\mathrm{D}_{2}$ : the para- $\mathrm{N}_{2}$ states consist of a quintuplet subsystem with $I=2$, whereas the ortho- $\mathrm{N}_{2}$ states are a triplet subsystem.

The electric field gradient asymmetry parameter $\eta$ was estimated according to diffraction data ${ }^{14}$ to be around 0.23 . Recorded ${ }^{14} \mathrm{~N}-\mathrm{NMR}$ spectra (Supplementary Fig. S2; right panel) do not show pronounced shoulder, expected for an $I=2$ quadrupolar powder pattern in absence of $m_{-2 \rightarrow-1}$ and $m_{1 \rightarrow 2}$ transitions. In fact, the spin system is well described by an $I=1$ spin system using the estimated value for $\eta$ (Supplementary Fig. S2; left panel).

According to structural data ${ }^{38}, r_{\mathrm{n}}$ can be estimated to be around $1.2 \AA$ at this pressure; four times longer than the thermal de-Broglie wavelength of a single ${ }^{14} \mathrm{~N}$ atom. Therefore, the wave function overlap should be negligible in molecular nitrogen at these pressures and nuclear spins considered unpaired. 
Table 1 Fitting parameters of ${ }^{1} \mathrm{H}-\mathrm{NMR}$ spectra.

\begin{tabular}{|c|c|c|c|c|c|}
\hline \multirow[b]{2}{*}{$P$ in GPa } & \multicolumn{2}{|c|}{ 1st Order quadrupole interaction } & \multicolumn{2}{|c|}{ Dipole-dipole interaction } & \multirow{2}{*}{$\begin{array}{l}\text { Lee-Goldburg decoupling } \delta_{\text {iso }} \\
\text { in ppm }\end{array}$} \\
\hline & $\mathrm{C}_{\mathrm{q}}$ in $\mathrm{kHz}$ & $\eta$ & $r_{n}$ in $\AA$ & $r_{n n}$ in $\AA$ & \\
\hline 20 & $28.1(6)$ & $0.44(6)$ & - & - & $8.665(112)$ \\
\hline 24 & $27.9(8)$ & $0.43(4)$ & - & - & $7.363(112)$ \\
\hline 36 & $30.0(7)$ & $0.50(7)$ & - & - & $6.429(112)$ \\
\hline 42 & $32.7(5)$ & $0.52(4)$ & - & - & $5.951(112)$ \\
\hline 47 & $35.2(6)$ & $0.44(6)$ & - & - & $5.928(125)$ \\
\hline 50 & $44.8(4)$ & $0.59(3)$ & - & - & $5.905(114)$ \\
\hline 54 & $48.0(3)$ & $0.46(7)$ & - & - & $5.924(150)$ \\
\hline 58 & $61.9(7)$ & $0.49(8)$ & - & - & $6.139(120)$ \\
\hline 68 & $43.9(9)$ & $0.37(9)$ & - & - & $6.670(173)$ \\
\hline 71 & $24.5(8)$ & $0.20(9)$ & $0.736(5)$ & $1.509(14)$ & $7.280(127)$ \\
\hline 77 & $18.6(7)$ & $0.15(7)$ & $0.733(5)$ & $1.457(13)$ & $8.479(195)$ \\
\hline 85 & $16.6(3)$ & $0.14(6)$ & $0.732(5)$ & $1.430(11)$ & $10.612(149)$ \\
\hline 97 & $15.0(4)$ & $0.10(5)$ & $0.732(5)$ & $1.340(12)$ & $15.351(100)$ \\
\hline 106 & $20.9(6)$ & $0.04(1)$ & $0.731(5)$ & $1.307(14)$ & $18.895(153)$ \\
\hline 115 & $19.2(9)$ & $0.05(7)$ & $0.729(5)$ & $1.270(11)$ & $21.323(147)$ \\
\hline 123 & $19.5(9)$ & $0.02(7)$ & $0.727(5)$ & $1.270(18)$ & $22.673(154)$ \\
\hline
\end{tabular}

$C_{\mathrm{a}}$ is the quadrupole coupling constant, $\eta$ the asymmetry parameter of the electric field gradient tensor in the principle axis system, $r_{\mathrm{n}}$ and $r_{\mathrm{nn}}$ are the nearest and second nearest neighbour distances, respectively. The isotropic chemical shift, $\delta_{\text {isor }}$ was derived after homonuclear Lee-Goldburg decoupling.

\section{Data availability}

The data supporting the findings of this study are publicly available from the corresponding author upon request.

\section{Code availability}

The custom Python code used for simulating the NMR spectra is available from the corresponding author upon request.

Received: 8 August 2020; Accepted: 6 November 2020;

Published online: 10 December 2020

\section{References}

1. Meier, T. At its extremes: NMR at giga-pascal pressures. Annu. Rep. NMR Spectrosc. 94, 1-74 (2017).

2. Meier, T. Journey to the centre of the Earth: Jules Vernes' dream in the laboratory from an NMR perspective. Prog. Nucl. Magn. Reson. Spectrosc. 106-107, 26-36 (2018)

3. Real, J. A., Gaspar, A. B. \& Carmen Muñoz, M. Thermal, pressure and light switchable spin-crossover materials. Dalton Trans. 2062-2079. https://doi.org/ 10.1039/b501491c (2005)

4. Rotaru, A. et al. Spin state dependence of electrical conductivity of spin crossover materials. Chem. Commun. 48, 4163-4165 (2012).

5. Antonangeli, D. et al. Spin crossover in ferropericlase at high pressure: A seismologically transparent transition? Science 331, 64-67 (2011).

6. Chekmenev, E. Y. Parahydrogen-induced magnetization of Jovian planets? ACS Earth Space Chem. 4, 495-498 (2020).

7. Mazin, I. I., Hemley, R. J., Goncharov, A. F., Hanfland, M. \& Mao, H. Quantum and classical orientational ordering in solid hydrogen. Phys. Rev. Lett. 78, 1066-1069 (1997).

8. Magdǎu, I. B., Balm, F. \& Ackland, G. J. Theory of high pressure hydrogen, made simple. J. Phys. Conf. Ser. 950, 042060 (2017).

9. Peña-Alvarez, M. et al. Quantitative rotational to librational transition in dense H2 and D2. J. Phys. Chem. Lett. 11, 6626-6631 (2020).

10. Silvera, I. F. The validity of ortho and para states of hydrogen at megabar pressures. J. Low. Temp. Phys. 112, 237-250 (1998).

11. Silvera, I. F. \& Pravica, M. G. Hydrogen at megabar pressures and the importance of ortho-para concentration. J. Phys. Condens. Matter 10 11169-11177 (1998).

12. Dias, R. P. \& Silvera, I. F. Observation of the Wigner-Huntington transition to metallic hydrogen. Science 355, 715-718 (2017).

13. Loubeyre, P., Occelli, F. \& Dumas, P. Synchrotron infrared spectroscopic evidence of the probable transition to metal hydrogen. Nature 577, 631-635 (2020)

14. Loubeyre, P. et al. X-ray diffraction and equation of state of hydrogen at megabar pressures. Nature 383, 702-704 (1996).
15. Labet, V., Gonzalez-Morelos, P., Hoffmann, R. \& Ashcroft, N. W. A fresh look at dense hydrogen under pressure. I. An introduction to the problem, and an index probing equalization of H-H distances. J. Chem. Phys 136, 074501 (2012).

16. Monacelli, L., Errea, I., Calandra, M. \& Mauri, F. Black metal hydrogen above $360 \mathrm{GPa}$ driven by proton quantum fluctuations. Nat. Phys. https://doi.org/ 10.1038/s41567-020-1009-3 (2020).

17. van de Bund, S. \& Ackland, G. J. Quadrupole arrangements and the ground state of solid hydrogen. Phys. Rev. B 101, 014103 (2020).

18. Cooke, P. I. C. et al. The raman signal of a hindered rotor. Phys. Rev. B $\mathbf{1 0 2}$ 064102 (2020).

19. Slichter, C. P. Principles of Magnetic Resonance (Springer, 1978).

20. Pake, G. E. Nuclear resonance absorption in hydrated crystals: fine structure of the proton line. J. Chem. Phys. 16, 327-336 (1948).

21. Ji, C. et al. Ultrahigh-pressure isostructural electronic transitions in hydrogen. Nature 573, 558-562 (2019).

22. Liu, X.-D., Howie, R. T., Zhang, H.-C., Chen, X.-J. \& Gregoryanz, E. Highpressure behavior of hydrogen and deuterium at low temperatures. Phys. Rev. Lett. 119, 065301 (2017).

23. Ackland, G. J. \& Loveday, J. S. Structures of solid hydrogen at 300 K. Phys. Rev. B 101, 094104 (2020).

24. Meier, T., Khandarkhaeva, S., Jacobs, J., Dubrovinskaia, N. \& Dubrovinsky, L. Improving resolution of solid state NMR in dense molecular hydrogen. Appl. Phys. Lett. 115, 131903 (2019)

25. Eremets, M. I. \& Trojan, I. A. Conductive dense hydrogen. Nat. Mater. 10, 927-931 (2011).

26. Mao, H. \& Hemley, R. J. Ultrahigh-pressure transitions in solid hydrogen. Rev Mod. Phys. 66, 671-692 (1994).

27. Strzhemechny, M. A. \& Hemley, R. J. New ortho-para conversion mechanism in dense solid hydrogen. Phys. Rev. Lett. 85, 5595-5598 (2000).

28. Meier, T. et al. Magnetic flux tailoring through Lenz lenses for ultrasmall samples: A new pathway to high-pressure nuclear magnetic resonance. Sci. Adv. 3, eaao5242 (2017)

29. Meier, T. et al. NMR at pressures up to 90 GPa. J. Magn. Reson. 292, 44-47 (2018)

30. Meier, T. et al. Pressure-induced hydrogen-hydrogen interaction in metallic FeH revealed by NMR. Phys. Rev. X 9, 031008 (2019).

31. Akahama, Y. \& Kawamura, H. High-pressure Raman spectroscopy of diamond anvils to $250 \mathrm{GPa}$ : Method for pressure determination in the multimegabar pressure range. J. Appl. Phys. 96, 3748 (2004).

32. Akahama, Y. \& Kawamura, H. Pressure calibration of diamond anvil Raman gauge to 310GPa. J. Appl. Phys. 100, 043516 (2006).

33. Howie, R. T., Gregoryanz, E. \& Goncharov, A. F. Hydrogen (deuterium) vibron frequency as a pressure comparison gauge at multi-Mbar pressures. $J$. Appl. Phys. 114, 073505 (2013).

34. Meier, T., Petitgirard, S., Khandarkhaeva, S. \& Dubrovinsky, L. Observation of nuclear quantum effects and hydrogen bond symmetrisation in high pressure ice. Nat. Commun. 9, 2766 (2018).

35. Bloembergen, N. \& Rowland, T. J. J. J. On the nuclear magnetic resonance in metals and alloys. Acta Met. 1, 731-746 (1953). 
36. Hughes, C. E. \& Harris, K. D. M. Calculation of solid-state NMR lineshapes using contour analysis. Solid State Nucl. Magn. Reson. 80, 7-13 (2016).

37. Harris, R. K. et al. Further conventions for NMR shielding and chemical shifts (IUPAC Recommendations 2008). Pure Appl. Chem. 80, 59-84 (2008)

38. Schiferl, D. et al. Structure of N2 at $2.92 \mathrm{GPa}$ and $300 \mathrm{~K}$. Acta Cryst. C 39, 1151-1153 (1983).

\section{Acknowledgements}

We thank Nobuyoshi Miyajima for help with the FIB milling. We are very thankful to Graeme Ackland and Gerd Steinle-Neumann for fruitful discussions. We thank the German Research Foundation (Deutsche Forschungsgemeinschaft, DFG, Project Nos DU 954/11-1, DU 393/13-1, DU 393/9-2, STE 1105/13-1 and ME 5206/3-1) and the Federal Ministry of Education and Research, Germany (BMBF, Grant No. 05K19WC1) for financial support. D.L. thanks the Alexander von Humboldt Foundation for financial support. M.P.-A. would like to acknowledge the support of the European Research Council (ERC) Grant "Hecate" reference no. 695527 secured by Graeme Ackland. N.D. thanks the Swedish Government Strategic Research Area in Materials Science on Functional Materials at Linköping University (Faculty Grant SFO-Mat-LiU No. 2009 00971).

\section{Author contributions}

T.M. and L.D. designed the experiment. T.M., S.K., A.K. and J.J. prepared the DACs and NMR resonators. T.M., D.L., M.P.-A., F.T. and A.K. performed and analysed the experiments. T.M., M.P.-A., D.L., F.T., N.D. and L.D. analysed the results and wrote the manuscript.

\section{Funding}

Open Access funding enabled and organized by Projekt DEAL.

\section{Competing interests}

The authors declare no competing interests.

\section{Additional information}

Supplementary information is available for this paper at https://doi.org/10.1038/s41467020-19927-y.

Correspondence and requests for materials should be addressed to T.M.

Peer review information Nature Communications thanks Takuo Okuchi, Michael Tayler and the other, anonymous, reviewer(s) for their contribution to the peer review of this work.

Reprints and permission information is available at http://www.nature.com/reprints

Publisher's note Springer Nature remains neutral with regard to jurisdictional claims in published maps and institutional affiliations.

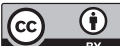

Open Access This article is licensed under a Creative Commons Attribution 4.0 International License, which permits use, sharing, adaptation, distribution and reproduction in any medium or format, as long as you give appropriate credit to the original author(s) and the source, provide a link to the Creative Commons license, and indicate if changes were made. The images or other third party material in this article are included in the article's Creative Commons license, unless indicated otherwise in a credit line to the material. If material is not included in the article's Creative Commons license and your intended use is not permitted by statutory regulation or exceeds the permitted use, you will need to obtain permission directly from the copyright holder. To view a copy of this license, visit http://creativecommons.org/ licenses/by/4.0/.

(C) The Author(s) 2020 